\title{
Antioxidant Potential of Rice Bran Oil Prepared from Red and White Rice
}

\author{
Dilini Bopitiya and Terrence Madhujith ${ }^{1 *}$ \\ Postgraduate Institute of Science \\ University of Peradeniya \\ Sri Lanka.
}

\begin{abstract}
The focus of the present study was to determine the total phenolic content $(T P C)$ and antioxidant potential of Rice Bran Oil (RBO). The bran of two rice varieties was obtained and stabilized and subsequently RBO was extracted into hexanes. The phenolic fraction of the oil was extracted into methanol by passing the oil through a glass column packed with silica ( $60 \AA$, pore diameter). TPC was determined and expressed as mg gallic acid equivalents (GAE) per gram extract. The antioxidant potential of the oil extracts was evaluated using DPPH and ABTS radical scavenging assays and $\beta$-carotene/linoleate model system. DPPH and ABTS radical scavenging capacities were expressed as $I C_{50}$ values and inhibition of linoleic acid induced oxidation of $\beta$-carotene was expressed as percent inhibition. The TPC of the two extracts was not significantly different $(p>0.05)$. However, the extract obtained from red rice variety exhibited significantly high $(p<0.05)$ DPPH radical scavenging activity and inhibition of linoleic induced $\beta$-carotene oxidation. It was also revealed that the radical scavenging activity was dose dependent. The RBO extracts did not exhibit any prooxidant activity at the highest level tested $(0.1 \mathrm{~g} / \mathrm{mL})$. The results revealed that the RBO possesses strong antioxidant activity compared to $\alpha$-tocopherol, thus, RBO can be categorized as an edible oil with high antioxidant potential.
\end{abstract}

Keywords: Antioxidants, $I C_{50}$ value, rice bran oil, total antioxidant capacity, total phenol content.

\section{INTRODUCTION}

Rice bran oil (RBO), also termed rice oil, is extracted from rice bran which is a by product of milling of Oryza sativa seeds. It mainly consists of pericarp and germ (Rukmini and Raghuram, 1991; Rubalya, et al., 2010; Elizabeth, 2011). RBO has optimal ratio of saturated fatty acids: monounsaturated fatty acids: polyunsaturated fatty acids of approximately $1: 2.2$ : 1.5 , which is very close to the recommendation of the World Health Organization. It mainly contains oleic (36-38\%), linoleic (35-38\%) and $\alpha$-linolenic (1.8-2.4\%) acids as unsaturated fatty acids and palmitic (21-25\%) and stearic (2.7 - 3.0\%) acids as saturated fatty acids (Kahlon et al., 1996; Gopala et al., 2005; Rubalya et al., 2010). It is also rich in various bioactive compounds including phytosterols, sterol esters, triterpene alcohols, gammaoryzanol, tocopherols, tocotrienols and other phenolic compounds. $\gamma$-Oryzanol is the major and unique component of RBO (Kahlon et al., 1996; Patel et al., 2004; Rebecca et al., 2005; Shahzad et al., 2006; Arab et al., 2011; Muntana et al., 2011).

\footnotetext{
1 Department of Food Science and Technology, University of Peradeniya, Peradeniya, Sri Lanka

* Corresponding author: madujith@yahoo.com
} 
Even though oils provide essential fatty acids and fat soluble vitamins, many epidemiological studies report that over consumption of edible oil leads to non communicable diseases (Shahidi et al., 1997; Philip et al., 2010; Rubalya and Neelamegam, 2012). Moreover, edible oils can easily become oxidized during processing and storage, thus producing a plethora of oxidative products which bring about oxidative stress-associated disease conditions such as atherosclerosis, diabetes mellitus, chronic renal failure, rheumatoid arthritis, cancer, and neurodegenerative diseases including Alzheimer's disease (Shahidi et al., 1997; Carrascopancorbo et al., 2005; Aleksandar et al., 2008; Katerina et al., 2010).

Edible oils which are rich in unsaturated fatty acids, especially Monounsaturated Fatty Acids (MUFA) are known to lower total and LDL cholesterol, triacylglycerol levels, and increase HDL cholesterol levels which help controlling blood pressure. Furthermore, they possess antithrombotic properties and also improve insulin sensitivity (Alessandra et al., 2007; Rubalya and Neelamegam, 2012). In addition, MUFA increase the stability of edible oils. The presence of bioactive components especially, phenolic antioxidants are attributable to better oxidative stability of oils. Moreover, the bioactive components present in edible oils reduce oxidative stress in the human body (Shahidi et al., 1997; Carrasco-pancorbo et al., 2005; Katerina et al., 2010; Rubalya and Neelamegam, 2012). Studies have shown several physiological effects related to these bioactive components. The bioactivities include cholesterol-lowering effects, pharmacological effects such as regulation of the oestrous cycle, growth-accelerating action, antitumor properties and the ability to promote skin capillary circulation and insulin sensitivity. In addition, the minor components present in edible oils contribute to improve texture and palatability and add characteristic flavours to food preparations (Shahidi et al., 1997 and Rubalya and Neelamegam, 2012).

RBO offers a wide range of applications that make it more acceptable among consumers in Asian countries such as Japan, Korea, China, Taiwan, Thailand, and Pakistan (Frank, 2005). $\mathrm{RBO}$ is generally considered to be one of the high quality vegetable oils in terms of its cooking attributes, shelf life, fatty acid composition and stability at higher temperatures. RBO enhances the taste and flavour of food items while reducing the absorption of oil during frying (Frank, 2005; Rebecca, et al., 2005; Shahzad, et al., 2006; Arab, et al., 2011; Muntana, et al., 2011). Furthermore, RBO is used in the manufacture of food products such as mayonnaise and salad dressings (Wasinee, et al., 2008). In addition, RBO improves skin tone and delays wrinkle formation, helps hair protection against premature graying and strengthen hair roots when topically applied. Therefore, RBO is used as an ingredient in many cosmetics (Frank, 2005).

RBO is a nutritionally superior oil compared to common plant oils. Several studies have demonstrated that RBO possesses anti-mutagenic activities (Frank, 2005; Shahzad, et al., 2006 \& 2011; Hemalatha and Ghafoorunissa, 2007; Yodmanee, et al., 2011). Rebecca, et al. (2005) showed that higher antihyperlipidemic action of RBO compared to soybean oil in several rat studies. Cicero and Derosa (2005) have identified RBO as an excellent source of PUFA, which lowers human blood cholesterol more effectively than sunflower, corn and safflower oils.

RBO shows an exceptionally high oxidative stability compared to soybean, palm, sesame, corn and most other popular vegetable oils. Several studies reported that RBO has high oxidative stability compared with that of soybean and rapeseed oils. Rubalya et al. (2010) exhibited that the thermal stability of RBO is greater than that of palm oil when subjected to repeated frying. The high oxidative stability of RBO makes it suitable for frying and baking applications (Frank, 2005). Rubalya and Neelamegam (2012) indicated that the oxidative 
stability of RBO was equivalent to or even better than that of soybean, corn, canola, cottonseed, and safflower oils in a model system that simulated deep frying conditions. Moreover, Arab et al. (2011) showed that the replacement of normal shortenings by different levels of RBO can produce superior quality cookies to prove effectiveness of RBO as a bakery shortening. In addition, Akiri et al. (2010) revealed that RBO based products have extended shelf life since RBO is extremely stable against rancidity and oxidative deterioration. Further, Gopala et al. (2005) reported that RBO help extend the shelf life of snack foods. The stabilized oil may be useful as spray oil for crackers, nuts, chips and other snack foods. However, despite the high content of MUFA, RBO remains as an underutilized oil in many countries.

Although a significant number of reports are available on the nutritional attributes of RBO, relatively little is reported in the scientific literature on its antioxidant potential. In this backdrop, the present study was carried out to quantify the total phenoilc content and evaluate the antioxidant potential of RBO.

\section{MATERIALS AND METHODS}

2, 2-Diphenyl-1-picrylhydrazyl hydrate (DPPH), Folin-Ciocalteu's phenol reagent, gallic acid, 2, 2'-azinobis (3-ethylbenzothiazoline-6-sulfonic acid) diammonium salt (ABTS), linoleic acid, $\beta$-carotene and Tween 20 were purchased from Sigma Chemicals USA. All other chemicals used were of analytical grade obtained from either Sigma or Himedia Chemicals. Rice samples: BG 400 (white) and LD 365 (red) varieties used in this study were purchased from the CIC Sales Centre, Kandy, Sri Lanka.

\section{Preparation of rice bran}

Two varieties of Sri Lankan raw rice, namely BG 400 (white) and LD 365 (red), were polished in a rice mill (Rice husker and polisher PM500, Japan). Rice bran samples were sieved through a $150 \mu \mathrm{m}$ sieve to remove unnecessary components such as paddy husk, broken grain parts and extraneous matter. So obtained bran was stabilized according to the method explained by Zogoneanu et al. (2008), by subjecting to microwave using a microwave oven (M-22.FP.04, Japan) for 3-5 min at $120{ }^{\circ} \mathrm{C}$ and cooled to room temperature overnight. This procedure was repeated three times to ensure complete stabilization. The stabilized rice bran samples were then vacuum packed in polyethylene bags and stored in the freezer at $-20{ }^{\circ} \mathrm{C}$ until used.

\section{Extraction of rice bran oil}

Oil of stabilized rice bran was extracted into hexanes using Soxhlet apparatus. The extraction was carried out on a water bath set at $50{ }^{\circ} \mathrm{C}$ for $8-9 \mathrm{~h}$ with $300 \mathrm{~mL}$ of n-hexanes. After extraction, the solvent was distilled off in vacuo at $45^{\circ} \mathrm{C}$. The extracted oil was stored at $-20{ }^{\circ} \mathrm{C}$ until further analysis.

\section{Extraction of phenolic fraction from rice bran oil}

The phenolic fraction present in $50 \mathrm{~g}$ oil was extracted into methanol by passing the oil through a glass column packed with silica $(60-\AA$ pore diameter) according to the method described by Steel et al. (2005). Hexanes and methanol (1:1) mixture was used for conditioning the column and hexanes and ethyl acetate (9:1) mixture was used for washing 
the column. The oil sample was dissolved in hexanes and introduced into the column and the phenoilc fraction was extracted into methanol. The extract was recovered after desolventizing in vacuo at $40{ }^{\circ} \mathrm{C}$. The prepared extracts were stored under frozen conditions at $-20^{\circ} \mathrm{C}$ after flushing with nitrogen gas. The frozen extracts were thawed and appropriately diluted before chemical analysis.

\section{Determination of total phenolic content}

The total phenolic content (TPC) of the crude RBO extracts was determined colorimetrically using Folin-Ciocalteu's reagent method as described by Kriengsak et al. (2006) with minor modifications. Oil extracts $(20 \mu \mathrm{L})$ were mixed with $100 \mu \mathrm{L}$ of Folin-Ciocalteu's reagent, left for $3 \mathrm{~min}$ and subsequently $300 \mu \mathrm{L}$ of sodium carbonate $(0.7 \mathrm{M})$ was added and vortexed. The absorbance of the resulting mixture was read at $725 \mathrm{~nm}$ using a UV visible spectrophotometer (UV 1601, Shimadzu, Japan) after leaving for $30 \mathrm{~min}$ at the room temperature $\left(25^{\circ} \mathrm{C}\right)$. The results were expressed as mg gallic acid equivalents (GAE) per litre of extract using gallic acid $(50-500 \mathrm{mg} / \mathrm{L})$ standard curve.

\section{Determination of DPPH radical scavenging capacity}

Antioxidant capacity of RBO extracts was assessed using 2,2-diphenyl-1picrylhydrazylhydrate (DPPH) free radical. The DPPH radical scavenging activity of oil extracts was quantified according to the method reported by Suwanna et al. (2010) with minor modifications. Five different concentrations ranging between 0.1 and $1.0 \mathrm{mg} / \mathrm{mL}$ of each extract $(0.5 \mathrm{~mL})$ was mixed with $2.5 \mathrm{~mL}$ of methanolic DPPH radical $(0.1 \mathrm{mM})$. After leaving for $20 \mathrm{~min}$ in the dark at room temperature, the absorbance was recorded at $517 \mathrm{~nm}$ using a UV visible spectrophotometer (UV 1601, Shimadzu, Japan). The radical scavenging activity was expressed as $\mathrm{IC}_{50}$ value that denotes the concentration of the sample required to scavenge $50 \%$ of DPPH radicals. $\alpha$-Tocopherol was used as the reference antioxidant.

\section{Determination of ABTS radical scavenging capacity}

The total antioxidant capacity of the extracts was determined using ABTS radical. The $\mathrm{ABTS}^{*+}$ was generated by reacting 2,2'-azobis (2-ethylbenzothiazoline-6-sulfonic acid) diammonium salt (ABTS) with 2,2'-azobis (2-methylpropanimidamide) dihydrochloride (AAPH), which acts as the radical generator. Five different concentrations ranging between 0.1 and $1.0 \mathrm{mg} / \mathrm{mL}$ of each extract $(40 \mu \mathrm{L})$ was mixed with $1.96 \mathrm{~mL}$ of ABTS radical solution and absorbance was measured at $765 \mathrm{~nm}$ using a UV visible spectrophotometer (UV 1601, Shimadzu, Japan). The radical scavenging activity was expressed as $\mathrm{IC}_{50}$ value that denotes the concentration of the sample required to scavenge $50 \%$ of ABTS radicals. $\alpha$ Tocopherol was used as the reference antioxidant (Robereta et al., 1999). 


\section{Determination of antioxidant efficacy using $\beta$-carotene/linoleate model system}

The ability of pomegranate extracts to inhibit $\beta$-carotene bleaching was estimated according to the method explained by Shahzad et al. (2006) with minor modifications. $\beta$ Carotene/linoleate emulsion was prepared by mixing $10 \mathrm{mg}$ of $\beta$-carotene, $40 \mathrm{mg}$ of linoleic acid, $600 \mathrm{mg}$ of Tween 20 emulsifier and $100 \mathrm{~mL}$ of oxygenated deionised water. The prepared emulsion $(2 \mathrm{~mL})$ was mixed with $200 \mu \mathrm{L}$ of extracts and incubated at $50{ }^{\circ} \mathrm{C}$. The oxidative loss of emulsion was monitored using a UV visible spectrophotometer (UV 1601, Shimadzu, Japan) at $470 \mathrm{~nm}$ wave length at every $15 \mathrm{~min}$ for $120 \mathrm{~min}$. The antioxidant activity (AA \%) was calculated according to the following equation and expressed as a percentage:

$$
\mathrm{AA}(\%)=\left\{\left[\left(\mathrm{AC}_{120}-\mathrm{AC}_{0}\right)-\left(\mathrm{AA}_{120}-\mathrm{AA}_{0}\right)\right] /\left(\mathrm{AC}_{120}-\mathrm{AC}_{0}\right)\right\} \times 100
$$

where, $\mathrm{AA}_{120}$ is the absorbance of the antioxidant at $120 \mathrm{~min}, \mathrm{AC}_{120}$ is the absorbance of the control at $120 \mathrm{~min}, \mathrm{AA}_{0}$ is the absorbance of the antioxidant at $0 \mathrm{~min}$, and $\mathrm{AC}_{0}$ is the absorbance of the control at $0 \mathrm{~min}$. $\alpha$-Tocopherol was used as the reference antioxidant.

\section{Determination of the dose dependence behaviour of activity}

Both red and white RBO extracts were tested for their DPPH and BATS radical scavenging activities at five different concentrations ranging from $0.01-0.1 \mathrm{~g} / \mathrm{mL}$. The DPPH and ABTS radical scavenging activities at each concentration was determined as explained above.

\section{Analysis of data}

All experiments were conducted in triplicate and complete randomized design was used. The statistical analysis was carried out using MS Office (Excel) and MINITAB version 16 software. The results are expressed as Mean $\pm \mathrm{SD}$ of three estimations.

\section{RESULTS AND DISCUSSION}

The quality of rice bran is mainly influenced by the presence of active lipase, which hydrolyses the triglyceride to fatty acids and glycerol (Yodmanee, et al., 2011). Therefore stabilization by microwave to deactivate this enzyme is essential.

\section{Total Phenolic Content (TPC)}

Phenolic constituents are one of the major group of compounds acting as primary antioxidants especially as free radical terminators (Oviasogie et al., 1999 and Marja et al., 2009). The total phenolic content of the methanolic extracts of crude RBO tested is given in Table 1. The TPC of extracts obtained from red RBO and white RBO was not significantly different ( $p>0.05$ ). Muntana et al. (2010) reported that the methanolic extracts of red, white, and black rice bran contained high TPC and black and white rice bran extracts showed similar TPC. The results ranged from 0.8931- $1.2239 \mathrm{mg}$ GAE/mg of bran. Shahzad et al. (2006) reported that TPC of four varieties of rice bran indigenous to Pakistan ranged from $251-359 \mathrm{mg} / 100 \mathrm{~g}$. 


\section{DPPH and ABTS radical scavenging activities}

DPPH and ABTS radical scavenging activities of the tested extracts are summarized in Table 1. The radical scavenging activities were expressed as $\mathrm{IC}_{50}$ value which is inversely proportional to the antioxidant activity (Kriengask et al., 2006 and Onder et al., 2009). According to the $\mathrm{IC}_{50}$ values, both $\mathrm{RBO}$ extracts possessed comparatively higher antioxidant activity than that of $\alpha$-tocopherol. The DPPH radical scavenging activity of red RBO extract was significantly $(\mathrm{p}<0.05)$ higher than that of white RBO extract. However, the ABTS radical scavenging activity of the two extracts were not significantly different. $(\mathrm{p}>0.05)$. Based on the $\mathrm{IC}_{50}$ values, both $\mathrm{RBO}$ can be categorized as oil with considerable antioxidant potential. Studies reported that the $\mathrm{IC}_{50}$ value of selected vegetable oil extracts, namely soy, sunflower, rapeseed, corn ranged from 29.7 to $34.0 \mu \mathrm{g} / \mathrm{mL}$ (Aleksander et al., 2008).

Table 1. TPC and antioxidant potential of extracts of red and white rice bran oil

\begin{tabular}{lllll}
\hline $\begin{array}{l}\text { Extract/Referenc } \\
\text { e antioxidant }\end{array}$ & $\begin{array}{l}\text { TPC } \\
(\mathrm{mg} \mathrm{GAE} / \mathrm{g} \\
\text { extract })\end{array}$ & $\begin{array}{l}\mathrm{DPPH} \\
\mathrm{IC}_{50} \mathrm{value} \\
(\mathrm{mg} / \mathrm{mL})\end{array}$ & $\begin{array}{l}\mathrm{ABTS} \mathrm{IC} \\
(\mathrm{mg} / \mathrm{mL})\end{array}$ & $\begin{array}{l}\text { \% inhibition of } \\
\beta \text {-carotene } \\
\text { oxidation }\end{array}$ \\
\hline $\begin{array}{l}\text { White rice bran } \\
\text { oil }\end{array}$ & $2.54^{\mathrm{a}} \pm 0.15$ & $0.027^{\mathrm{b}} \pm 0.007$ & $0.018^{\mathrm{a}} \pm 0.006$ & $72.74^{\mathrm{b}} \pm 0.02$ \\
$\begin{array}{l}\text { Red rice bran oil } \\
\text { a-Tocopherol }\end{array}$ & $2.63^{\mathrm{a}} \pm 0.20$ & $0.025^{\mathrm{a}} \pm 0.001$ & $0.017^{\mathrm{a}} \pm 0.001$ & $79.12^{\mathrm{c}} \pm 0.00$ \\
& n.a. & $0.031^{\mathrm{c}} \pm 0.004$ & $0.046^{\mathrm{b}} \pm 0.007$ & $45.03^{\mathrm{a}} \pm 0.03$
\end{tabular}

na. - not applicable; values sharing the same superscript in the same column are not significantly different. Values are means of three determinations expressed as Mean $\pm S D$.

\section{Inhibition of linoleic acid induced $\beta$-carotene oxidation}

$\beta$-Carotene is highly vulnerable for autoxidation which can be triggered by linoleic acid. The $\beta$-carotene/linoleate model system has been used as an important assay to assess the efficacy of antioxidative constituents in inhibition of linoleic acid induced oxidation of $\beta$-carotene. The inhibition of degradation of $\beta$-carotene as expressed by percent discoloration of RBO extract and $\alpha$-tocopherol at $2.0 \%$ (w/v) concentration is illustrated in Table 1. Both RBO extracts were capable of effectively retarding the oxidation of $\beta$-carotene in the emulsion system compared to the reference antioxidant, $\alpha$-tocopherol (Fig. 1). Red RBO extract showed significantly high $(\mathrm{p}<0.05)$ inhibition than white RBO extract. 


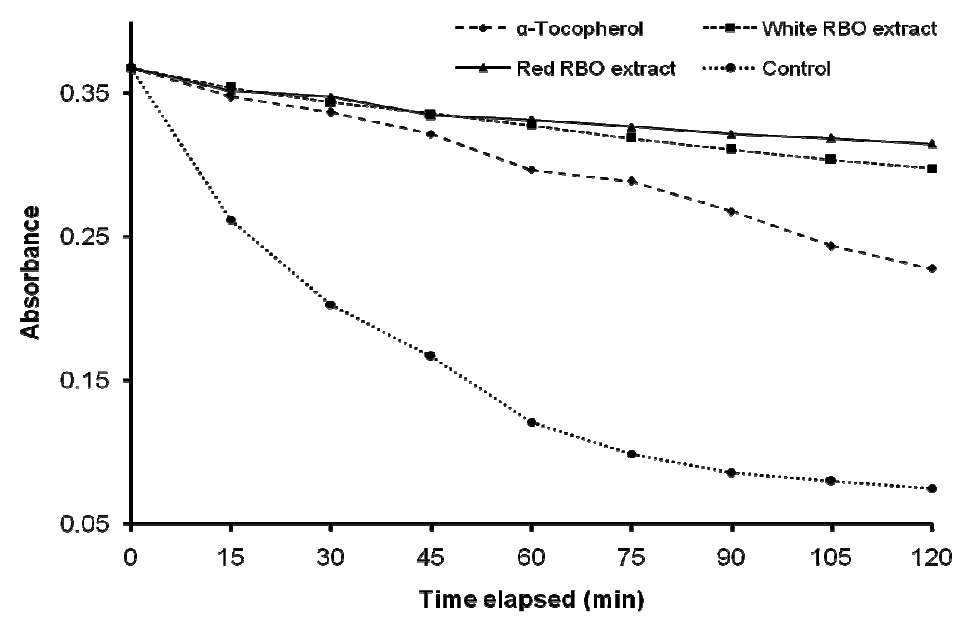

Fig. 1. Decrease of absorbance in the emulsion system containing $\beta$-carotene-linoleic acid-extract or reference antioxidant over $120 \mathrm{~min}$.

\section{Dose dependant behaviour of RBO extracts}

As shown in Fig. 2 and 3, the inhibitory effect of the phenolic extracts on DPPH and ABTS radicals followed a dose-dependent manner within the concentration tested $(0.01-0.1$ $\mathrm{g} / \mathrm{mL}$ ). Both red and white RBO extracts tested showed a strong correlation with the concentration and DPPH and ABTS radical scavenging activities. The radical scavenging activity increased sharply with increasing concentration of the sample extract. Therefore, it was evident that the concentration of the RBO extracts strongly influenced the antioxidant power. Furthermore, no prooxidant activity of the extracts was observed even at the highest concentration tested $(0.1 \mathrm{~g} / \mathrm{mL})$.

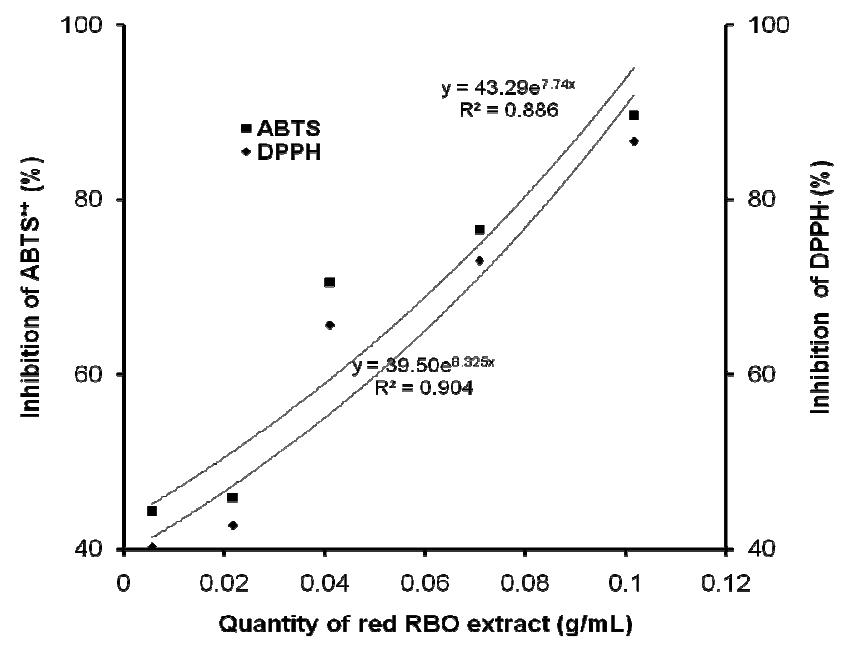

Fig. 2. Correlation between the quantity of the red RBO extract and percent inhibition of $\mathrm{ABTS}^{*+}$ and DPPH* 


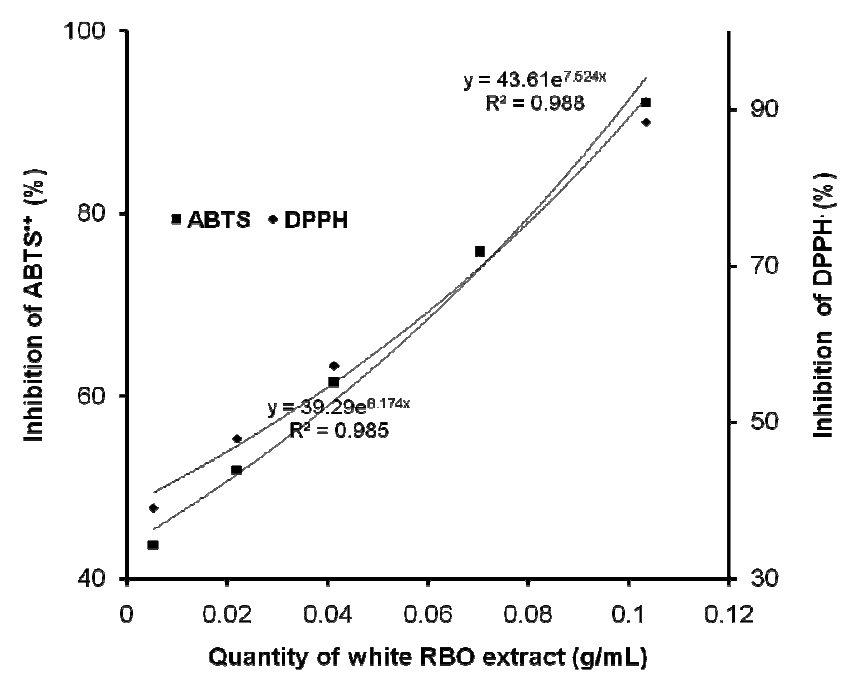

Fig. 3. Correlation between the quantity of white RBO extract and percent inhibition of $\mathrm{ABTS}^{*+}$ and DPPH*

In summary, the antioxidant activity as measured by DPPH and ABTS radical scavenging efficacy and inhibition of oxidation of $\beta$-carotene of white and red RBO was not significantly ( $p>0.05)$ different. Results revealed that both white and red RBO extracts possessed strong antioxidant activity compared to the reference antioxidant, $\alpha$-tocopherol, thus, RBO can be categorized as an edible oil with high antioxidant potential. Antioxidant activity as measured by ABTS and DPPH radical assays and using $\beta$-carotene/linoleate model system well correlated with TPC $(0.972 \leq \mathrm{r} \leq 0.999)$. The results indicated that antioxidant activities of RBO extracts were largely attributable to the phenolic compounds present. Therefore, RBO can be considered a potential source of antioxidative constituents thus can be a useful ingredient in preparation of food.

\section{CONCLUSIONS}

The antioxidant activity of methanolic white and red RBO extracts as measured by DPPH and ABTS radical scavenging potential and inhibition of degradation of $\beta$-carotene was significantly higher than that of the reference antioxidant, $\alpha$-tocopherol. RBO can be categorized as an edible oil with strong antioxidant potential. The antioxidant activities of the RBO extracts are largely attributable to the phenolic compounds present in RBO. It can also be concluded that the antioxidant activity as measured by ABTS and DPPH radicals is dose dependant with no prooxidant activity even at the highest level tested.

\section{ACKNOWLEDGMENTS}

The financial support from National Research Council, Sri Lanka (NRC grant 0945) is gratefully acknowledged. 


\section{REFERENCES}

Akiri S.V.C.R., Sareddy G.R., Phanithi P.B. and Attipalli, R.R. (2010). The antioxidant and antiproliferative activities of methanolic extracts from Njavara rice bran, BMC Complementary and Alternative Medicine. 10(4), 1 - 9.

Aleksander S., Malgorzata, N. and Elenora L. (2008). The content and antioxidant activity of phenoilc compounds in cold pressed plant oils. Journal of Food Lipids. 137 - 149.

Arab, F., Alemzadeh, I. and Maghsoudi, V. (2011). Determination of antioxidant component and activity of rice bran extract, Scientia Iranica. 18(6), 1402 - 406.

Bendini, A., Cerretani, L., Vecchi, S., Carrasco-Pancorbo A. and Lercker, G. (2007). Protective effects of extra virgin olive oil phenolics on oxidative stability in the presence or absence of copper ions, J. Agric. Food Chem. 54(2007), 4880 - 4887.

Carrasco-pancorbo, A., Cerretani, L., Bendini, A., Segura-carrentero, A., Del Carlo, M., Gallina-toschi, T., Lercker, G., Compangnone, D. and Ndez-gutiea, F. (2005). Evaluation of the antioxidant capacity of individual phenolic compounds in virgin olive oil, J. Agric. Food Chemistry. 53, 8918 - 8925.

Cicero, A. F. G. and Derosa, G. (2005). Rice bran and its main components, potential role in the management of coronary risk factors, Current topics in Nutraceutical research. 3(1), 29 46.

Elizabeth, P.R. (2011). Bioactive food components and health properties of rice bran, Timely Topics in Nutrition JAVMA, 238(5), 593 - 600.

Frank, T. (2005). Rice bran oil. In F. Shahidi (6 $6^{\text {th }}$ ed.) Bailey's Industrial oil and fat products, John Wiley \& Sons, Inc. 465 - 489.

Gopala, K.A.G., Khatoon, S. and Babylatha, R. (2005). Frying performance of processed rice bran oils. Journal of Food Lipids. 12, 1 - 11.

Hemalatha, S. and Ghafoorunissa. (2007). Sesame lignans enhance the thermal stability of edible vegetable oils. Food Chemistry. 105, 1076 - 1085.

Kahlon, T.S., Chow, F.I., Chiu, M.M., Hudson, C.A. and Sayre, R.N. (1996). Cholesterollowering by rice bran and rice bran oil unsaponifiable matter in hamsters, Cereal Chem. $73(1), 69-74$.

Katerina, S. Miniotia and Georgioua, C.A. (2010). Comparison of different tests used in mapping the Greek virgin olive oil production for the determination of its total antioxidant capacity. Grasa Y Aceites. 61(1), 45 - 51.

Kriengsak, T., Unaroj, B. Kevin, C., Luis, C. and David, H.B. (2006). Comparison of ABTS, DPPH, FRAP, and ORAC assays for estimating antioxidant activity from guava fruit extracts. Journal of Food Composition and Analysis. 19, 669 - 675. 
Marja, P.K., Anu, I.H., Heikki, J.V., Jussi-pekka, R., Kalevi, P., Tytti, S.K. and Marina, H. (1999). Antioxidant activity of plant extracts containing phenolic compounds. Journal of Agriculture and Food Chemistry. 47, 3954 - 3962.

Muntana, N. and Prasong, S. (2010). Study on total phenolic contents and their antioxidant activity of Thai white, red and black rice bran extracts, Pakistan Journal of Biological Sciences. 13(4), 170 - 174.

Onder, K., Sezai, E., Memnune, S., Celi, T. and Sedat, S. (2009).Total phenolics and antioxidant activity of jujube genotypes selected from Turkey. African Journal of Biotechnology. 8(2), 303 - 307.

Oviasogie, P.O., Okoro, D. and Ndiokwere, C.L. (2009). Determination of total phenolic amounts of some edible fruits and vegetables. African Journal of Biotechnology. 8(12), 2819 - 2820.

Patel, M. and Naik, S.N. (2004). Gamma-oryzanol from rice bran oil: A review, Journal of Scientific and Industrial Research. 63, 569-578.

Philip, J.K., Joseph, Z.B., Kanu, J.B. and Kandeh, J.B.A. (2010). Nutraceutical importance of sesame seed and oil: A review of the contribution of their lignans. Journal of Biomedical Research. 2(1), 4 - 6.

Rebecca, K., Gopinath, N., Mario, V. and Anura, V.K. (2005). Use of rice bran oil in patients with hyperlipidaemia, The National Medical Journal of India. 18(6), 292 - 296.

Robereta, R., Nicoletta, P., Anna, P., Ananth, P., Min, Y. and Catherine, R. (1999). Antioxidant activity applying an improved ABTS radical cation decoloration assay. Journal of Free Radical Biology and Medicine. 26 (9/10), 1231 - 1237.

Rubalya, V. S. and Neelamegam, P. (2012). Antioxidant potential in vegetable oil, Research Journal of Chemistry and Environment. 16(2), 87 - 94.

Rubalya, V.S., Arockia, S.P. and Angelin, P.A. (2010). Antioxidant stability in palm and rice bran oil using simple parameters, Rasayan Journal of Chemistry. 3(1), 44 - 50.

Rukmini, C. and Raghuram, C.T. (1991). Nutritional and the biochemical aspects of the hypolepidemic action of rice bran oil: review, Journal of the American College of Nutrition. 10(4), 593 - 601.

Shahidi F., Wanasundara P.K.J.P.D. and Wanasundara U.N. (1997) Changes in edible fats and oils during processing. Journal of food lipids. 4(19), 9 - 23.

Shahzad, A.S.C., Abdullah, I.H., Muhammad, Z. and Muhammad, K.K. (2011). Analytical characterization of rice (Oryza sativa) bran and bran oil from different agro-ecological regions, Pakistan Journal of Agricultural Science. 48(3), 243 - 249.

Shahzad, A.S.C., Farooq, A. Maleeha, M. and Jawad-ur-Rehman, B. (2006). Evaluation of the antioxidant activity of rice bran extracts using different antioxidant assays, Grasas Aceites. 57(3), 328 - 335. 
Suwanna, V., Chittima, M., Lukkhana, .I, Wikalda, S. and Narisa, K. (2010). Examination of antioxidant activity and development of rice bran oil and gamma-oryzanol microemulsion, Journal of Health Research. 24(2), 67 - 72.

Steel, C.J., Dobarganes, M.C. and Barrera-Arellano, D. (2005). The Influence of natural tocopherols during thermal oxidation of refined and partially hydrogenated soybean oils. Grasas Aceites. 56, 46 - 52.

Wasinee, K., Ketmanee, W. and Artiwan, S. (2008). Value added products from by-products of rice bran oil processing, Chiang Mai Journal of Science. 35(1), 116 - 122.

Yodmanee, S., Karrila, T.T. and Pakdeechanuan, P. (2011). Physical, chemical and antioxidant properties of pigmented rice grown in southern Thailand, International Food Research Journal. 18(3), 901 - 906.

Zogoneanu, I.G., Williams, L., Xu, Z. and, C.M. (2008). Determination of antioxidant components in rice bran oil extracted by microwave-assisted method, Bioresource Technology. 99 (11), 4910 - 4918. 